\title{
EL ENTOMÓLOGO ECONÓMICO: ENTREVISTA CON ALAN KIRMAN"
}

\author{
David Bassett \\ François Claveau
}

\begin{abstract}
A lan P. Kirman (Londres, 1939) es profesor honorario en Al'Université D'Aix-Marseille III e investigador en GREQAM (Groupe de Investigación en Economía Cuantitativa D'Aix-Marsei1le). Ha publicado más de cien artículos académicos y ha sido autor de muchos libros que incluyen notables monografías sobre análisis de equilibrio general (Hildenbrand y Kirman 1976; 1988) y más recientemente de Economia compleja: racionalidad individual y colectiva (Kirman 2011, revisado en este número de EJPE).

La obra del profesor Kirman toca muchos temas de interés para metodólogos económicos y por ello nos encantó tener la oportunidad de entrevistarlo cuando visitó el Instituto Erasmo para la Filosofía y la Economía (EIPE) a finales de noviembre de 2010 para presentar un escrito sobre el estado de macroeconomía.
\end{abstract}

"DOI: https://doi.org/10.18601/01245996.v21n40.13. Tomada de Erasmus Journal for Philosophy and Economics, 2(4), pp. 42-66. Traducción de Alberto Supelano. Se publica con las autorizaciones correspondientes. Recepción: 04-09-2018, aceptación: 30-10-2018. Sugerencia de citación: Basset, David y Claveau, F. (2019). El entomólogo económico: Entrevista con Alan Kirman. Revista de Economía Institucional, 21(40), 343-366.

a Estudiante de la Maestría en Investigación en filosofía y economía, Facultad de Filosofía, Erasmus University, Rotterdam, [d.e.bassett@bham.ac.uk], [https://orcid.org/0000-0002-7529-0829].

b Candidato a Doctor, Instituto Erasmus de Filosofía y Economía (EIPE), y coeditor del Erasmus Journal for Philosophy and Economics, [francois.claveau@ USherbrooke.ca], [https://orcid.org/0000-0001-7129-7794]. 
En esta entrevista el profesor Kirman analiza su comprensión de la relación entre comportamiento individual y patrones agregados, por qué es esencial considerar las interacciones entre agentes, y qué nos puede enseñar el estudio del comportamiento de las hormigas sobre las acciones humanas colectivas. Explica los conceptos básicos de su enfoque "interaccionista", incluidos los microfundamentos, la racionalidad y la emergencia, y reflexiona sobre el potencial de la modelación basada en agentes, las limitaciones de la teoría de juegos, la posibilidad del análisis a nivel agregado y la relevancia de los estudios del comportamiento. La entrevista también cubre un campo más amplio: discute los diferentes objetivos de la economía (p ej., explicar, predecir y controlar), el papel de las matemáticas en la economía moderna y el estado de macroeconomía.

EJPE: ¿Que lo llevó a la economía y cómo describiría su investigación en los primeros años de su carrera?

ALAN KIRMAN: Bien, mi historia es un poco extraña porque empecé después de mi primer grado en Oxford como profesor de geografía escolar. Pero me pregunté: “¿Quiero hacer esto el resto de mi vida?" Fui a algunas clases nocturnas de economía organizadas por la Workers Educational Association, y pensé: “Oh, eso es realmente interesante. Quizá debería intentar y hacer algo con esto.”

Primero hice un año de diplomado (parte de él en economía internacional) en la Johns Hopkins School de Estudios Internacionales en Bolonia (Italia). Una de las personas que enseñaba allí, Ira Scott, me recomendó que hiciera un doctorado en Minnesota, así que fui a Minnesota el año siguiente. Allí, era muy frío y sumamente matemático. Pero no había cursado matemáticas antes, y mi consejero Hugo Sonnenschein me dijo que también debía graduarme en matemáticas.

En la primavera pensé que eso no era lo qué había ido a hacer allí. Para mí, la economía trataba del desempleo y la inflación, etc., pero allí estaba luchando con teoremas de punto fijo y todo eso. Le dije a Hugo: “Mira, ¿por qué no puedo hacer estas otras cosas?” É1 dijo: “No, no, eso es macroeconomía, y la macroeconomía es sobre sabiduría. La microeconomía es sobre análisis, y los jóvenes deberían hacer micro".

De todos modos, conseguí una beca para ir a Princeton, donde pensé que iba a hacer economía más real. Una vez allí observé a las personas que estaban enseñando y de lejos el profesor más interesante e inspirador era Harold W. Kuhn, quien -infortunadamente para mí- era iprofesor de matemáticas y también de economía! De modo 
que hice mi tesis con él, aplicando teoría de juegos no cooperativos al comercio internacional.

Después pasé al equilibrio general. También trabajé en muchas otras cosas en mis primeros años, porque, ya sabes, el equilibrio general no es muy inspirador. Quiero decir, es un gran juego intelectual, pero es tan matemático. Así que me dediqué a la justicia, la elección social, un poco al comercio internacional; muchas cosas diferentes, nada muy profundo, y así es cómo empecé.

${ }_{\dot{¿}} N o s$ puede dar una breve historia "interna" del creciente descontento con el proyecto de equilibrio general; del papel de los resultados de SonnenscheinMantel-Debreu, por ejemplo?

Los resultados de Sonnenschein (1972), Mantel (1974) y Debreu (1974) tuvieron un gran impacto. En su libro La demanda del mercado, Werner Hildenbrand (1994) dijo que solía trabajar bajo el supuesto de que la fundamentación microeconómica nos daría predicciones a nivel agregado, pero estos resultados derrumbaron eso.

Supimos que más o menos sale cualquier cosa de lo que podemos observar a nivel agregado. Por ejemplo, cómo se puede hacer estática comparativa en macroeconomía si se tienen varios equilibrios? ¿Con qué equilibrio se comienza y a cuál se va? Ese es un problema. Además, si no se tiene ni idea de si las economías que comienzan en equilibrio llegarán realmente al equilibrio, ¿por qué estar tan interesados en el equilibrio? Michio Morishima (1984) dijo que tal concepto sería de interés puramente intelectual.

Ese es el tipo de cosas que hacían sentir incómodas a las personas por las condiciones muy restrictivas que normalmente imponemos a los individuos. Cuándo se aprende que incluso con esas condiciones no se va a tener mucha estructura en el agregado, uno se empieza a decir a sí mismo que tal vez este proyecto tenga algún tipo de debilidad fundamental. Muchos microeconomistas dijeron que esto no era muy bueno, pero los macroeconomistas no aceptaron ese mensaje. Simplemente dijeron que tendremos que simplificar las cosas hasta que lleguemos a una situación en la que tengamos unicidad y estabilidad. Y entonces, por supuesto, llegamos al famoso individuo representativo.

Hildenbrand adoptó una posición radical y dijo que deberíamos olvidarnos de toda esta optimización individual y simplemente observar las distribuciones de cómo se comportan las personas y ver si podemos extraer reglas sobre la economía. ¡Eso cayó como un globo de plomo! Él dio charlas en Berkeley y en todas partes y la gente fue 
sumamente hostil. De modo que su enfoque nunca despegó y, sin embargo, pienso que es una manera muy positiva de hacer las cosas.

Su libro más reciente se llama Economia compleja (Kirman 2011). Muchos economistas de los últimos veinte años o algo asi han respaldado etiquetas similares. ¿Qué se considera "complejidad"? ¿Cómo pasó del equilibrio general a este otro proyecto?

E1 Journal of Mathematical Economics se publicó por primera vez en 1974. Hans Föllmer -matemático en Bonn en ese momento, después en Zürich y ahora en Berlín- publicó un artículo en el primer número de esa revista que se llamaba "Economías aleatorias con muchos agentes interactuantes”(Föllmer 1974). Allí mostró que si hay muchas personas que tienen sus preferencias regulares y así sucesivamente, pero esas preferencias están influidas por sus vecinos -como las partículas en el modelo Ising de la física- eso podía derrumbar la noción subyacente de un equilibrio único o, en términos alternativos, que no se podía decir mucho sobre el agregado una vez había esta interacción.

Empecé a pensar en modelos de interacción y hablé con Hans sobre eso pero no fui suficientemente imaginativo. Luego conocí a un matemático de Warwick llamado David Rand y tuvimos una larga discusión sobre si podíamos pensar en la demanda de manera diferente, considerando que la demanda de cada individuo era influida por las demandas de sus "vecinos".

Creo que entonces comencé a pensar en sistemas en los que se tienen estos individuos realmente primitivos interactuando. Eso es lo que llegó a ser la base de mi visión de la complejidad: muchos individuos bastante simples que por sus interacciones generan fenómenos a nivel agregado que no coinciden con lo que se ve en el nivel inferior. En economía normalmente tomamos un atajo: simplemente suponemos que lo que ocurre allí arriba se parece a lo que sucede aquí abajo.

El término "microfundamentos" es muy popular en economía. Diría usted que su enfoque es crítico de la búsqueda de microfundamentos o que sólo es crítico de los microfundamentos especificos que están de moda en economía?

Depende de lo que quiera decir exactamente por microfundamentos; ¿quiere decir con eso que deseo reducir la economía en algún sentido a observar algún tipo de "individuo típico o representativo", de modo que una vez entienda cómo funciona un individuo, entonces entiendo cómo funciona la economía? Bien, entonces soy crítico (ver Kirman 1992). También soy crítico de los microfundamentos particulares que usamos. ¿Por qué debemos enfocarnos tan estrechamente en 
estos axiomas de racionalidad que hemos impuesto? Esos son los dos aspectos en los que sería crítico.

Esto no significa que no se deba interesar en lo que hacen los individuos, después de todo, de eso se trata la modelación basada en agentes. También le puede interesar cómo afecta el comportamiento individual el funcionamiento de la economía pero no se debe tener una idea simple, aditiva y mecánica de esta transición.

En alguna parte de mi nuevo libro (Kirman 2011,19) hay una cita de dos neurólogos, Churchland y Sejnowski (1992,4). Ellos dicen que la agregación en neurociencia es mucho más complicada que sumar simplemente las características de las neuronas. Esto no debería impedir que le interese lo que está sucediendo a nivel neuronal pero, si quiere entender las actitudes de las personas y lo que piensan, no va a limitarse a examinar las neuronas, ¿cierto? Le interesará saber qué le sucede a la red de neuronas: cómo se combinan y cuáles se disparan cuando se tienen ciertos estados de ánimo, etc.

Usted rechaza los supuestos de la racionalidad estándar en economia. ¿Cuáles son sus razones son para ello? ¿Cuáles son los substitutos?

Esos axiomas -y puede encontrar toda una serie de personas desde Pareto en adelante que expresan el mismo argumento- provienen de la introspección de los economistas y de lo que creen que es necesario para su trabajo, no de la observación de lo que hacen las personas.

Algunos de estos axiomas parecen naturales, al menos a primera vista. Por ejemplo, la transitividad parece una idea natural: si se prefiere $\mathrm{A}$ a $\mathrm{B}$ y B a C, también se prefiere $\mathrm{A}$ a $\mathrm{C}$. Pero si mira atentamente cómo definen los economistas las cosas sobre las que se toman decisiones, nunca podrá observar si un individuo toma o no decisiones transitivas.

Mi problema principal es que ninguno de estos axiomas se adopta observando a muchas personas. En otras disciplinas, eso es lo que se hace. Se observa y luego se intenta desarrollar un modelo que pueda explicar lo que se observa. En economía, empezamos haciendo la formalización y construyendo modelos que sean internamente coherentes pero a menudo muy alejados de la realidad. Para construir modelos que podamos analizar formalmente, necesitamos hacer algunos supuestos formales. Como dije, muchos académicos que empezaron con Pareto hicieron básicamente el mismo comentario: estos supuestos no son naturales, no se refieren a lo que hacen las personas, sino a lo que necesitamos para proseguir nuestro análisis. Esa es mi objeción real. 
¿Con qué los remplaza? ¿Acaba de decir que las personas solo toman decisiones aleatorias arbitrarias? Bueno, por supuesto que no. $\mathrm{El}$ argumento que expondría sería que, en algún sentido, las personas ven direcciones en las que piensan que su bienestar mejora, e intentan moverse en esas direcciones. Una manera simple de modelar esto es dar a los agentes reglas simples que se encuentren plausibles y luego ver cómo funciona eso. En tal modelo, las personas no son irracionales, pero la racionalidad debe tener una definición mucho más abierta.

Usted muestra cierta simpatía hacia el proyecto de economía del comportamiento para proporcionar supuestos psicológicamente refinados sobre los agentes económicos. Al mismo tiempo, afirma que tenemos mucho que aprender de los estudios de hormigas, abejas y otros animales sociales que se pueden modelar como acciones basadas en reglas simples de comportamiento. Estas dos lineas de investigación, que refinan la psicología de nuestros agentes modelados y buscan reglas simples de comportamientos, no están en tensión?

La distinción es realmente la siguiente. Digamos que observo que las personas se comportan de algunas maneras y que en conjunto generan un patrón agregado. Me digo a mí mismo: “¿Puedo pensar este fenómeno en términos de las reglas que siguen las personas sin preocuparme por el momento por sus intenciones?". Eso es como el fenómeno de las hormigas, en el sentido de que las hormigas interactúan de maneras muy simples.

Ahora usted podría decir que está interesado en por qué actúan así. ¿Por qué siguen estas reglas? Se puede decir que la evolución las ha llevado a seleccionar las reglas que siguen. Esto es lo que a menudo se dice de las hormigas. Sin embargo, Deborah Gordon, una entomóloga famosa recopiló abundante evidencia para mostrar que las hormigas suelen ser individualmente ineficientes y no hacen lo que intentan hacer (ver Gordon 2010). Aunque logran mucho, no parecen comportarse de manera óptima en un sentido estándar. Creo que los economistas han aceptado con demasiada facilidad los argumentos evolutivos poco sofisticados. Su consejo a las personas que argumentan en favor del comportamiento óptimo es "dedicar tiempo a observar a las hormigas". Se podría dar el mismo consejo a los economistas, “jobservar a los agentes económicos!”. Así, cuando observamos a los seres humanos quizá deseemos saber mucho más de que, en general, parecen seguirlas reglas simples y ver el aspecto psicológico de las cosas. Por esto me interesé en la neuroeconomía 
y publiqué un par de artículos sobre eso (p, ej., Oullier et al. 2008; Kirman y Teschl 2010).

De modo que no creo que haya una contradicción. Cuando nos interesamos en los humanos no sólo nos interesa saber qué reglas siguen sino también por qué siguen estas reglas particulares y por qué a menudo parecen comportarse de manera no racional. Esta sería mi distinción.

Usted ha hecho muchas contribuciones a la difícil pregunta de cómo conectar macro patrones con el comportamiento de los agentes. ¿Cuáles son los principales resultados a este respecto? Por ejemplo, puede contarnos sobre su fascinante trabajo acerca de los mercados de pescado?

Hay dos resultados importantes en la relación entre los patrones agregados y el comportamiento individual. En primer lugar, aunque los individuos no se "comporten bien" en el sentido estándar, el agregado puede tener un comportamiento bastante ordenado. En segundo lugar, también es posible que cuando tenemos muchos individuos bien comportados, el agregado parezca ser mucho más desordenado que el comportamiento de los individuos.

Al considerar la primera posibilidad, allí es donde voy a observar mirar los mercados reales -como los de pescados (Härdle y Kirman 1995; Kirman 2011, cap. 3)- e intento comprender qué está sucediendo. En los mercados, los individuos están en situaciones muy complicadas; conocen mucha información de todas las personas que los rodean, y suceden varias cosas que usualmente no se incorporan en nuestros modelos.

Se podría intentar y modelar esto como un juego enorme y complicado. Pero, de hecho, estas personas se conforman con hacer cosas que realmente son bastante simples. Por ejemplo, muchos ellos se vuelven sumamente leales; siempre compran a un vendedor. Una vez se ha establecido ese hecho, entonces se puede empezar a ver una especie de patrón emergiendo en el mercado. Algunas personas andan de compras, pero en conjunto se obtiene una relación negativa bastante buena (que para ser precisos no se debería llamar "demanda") entre la cantidad de pescado en el mercado y los precios obtenidos. Los patrones simples de comportamiento individual realmente generan a nivel agregado algo que se parece bastante a lo que se podría haber pensado como una función de exceso de demanda agregada clásica, pero ciertamente no se deriva de que cada individuo tenga su propia función de exceso de demanda regular. (A propósito, ¡en economía históricamente ha habido mucha discusión sobre si lo que observamos 
cuando las personas hacen compras corresponde a la definición formal de demanda! Pero esa es una discusión para otro día.)

Considerando la segunda posibilidad, si se observan los mercados financieros (Kirman 2011, cap. 11), las personas que parecen seguir reglas que en algún sentido son racionales a veces puede generar grandes movimientos en el mercado, no porque de repente se vuelvan irracionales sino porque tienen en cuenta lo que están haciendo otras personas y comienzan a seguir la tendencia. Las cascadas de información y este tipo de fenómenos surgen no porque las personas sean simplemente raras. Suceden porque las personas interactúan entre sí. Esta interacción puede llevar a movimientos masivos que no se podrían haber previsto si solo se observara a los individuos en aislamiento.

Así se obtienen dos cosas: comportamiento "malo", "irracional” o "extraño" que en cierto modo se suaviza, y lo contrario.

El término "racionalidad colectiva" aparece en el subtitulo de su libro reciente. ¿Qué quiere decir con eso? Cómo se relaciona con nuestra comprensión usual de la racionalidad individual?

Bien, no estoy feliz con "racionalidad". Uno de los problemas que encontramos es que las personas hoy han absorbido de algún modo la noción de racionalidad del economista, así que cuando las personas dicen "racional", inmediatamente tienen en mente algo como lo que los economistas definen como racionalidad. De hecho, la racionalidad se puede pensar de muchas maneras.

Para mí, racionalidad significaría algo más que un comportamiento coherente o interpretable: un comportamiento que no es exactamente aleatorio. Entonces "racionalidad colectiva" significaría que, en algún sentido, este grupo o sociedad se mueve de una manera que se puede observar y anticipar y parece tener un propósito; aunque no quiero insistir demasiado en "tener propósito" porque no es claro que el agregado tenga propósitos.

En este sentido, "racionalidad colectiva" bien se podría aplicar a un sistema físico, donde claramente no hay ninguna intención involucrada. Tomemos un sistema formado por partículas físicas (ver Kirman 2011, cap. 6; y Vinkovi y Kirman 2006). La tendencia básica del sistema es minimizar su energía total. Se podría querer decir que el sistema intenta reducir su energía, pero no es intencional. El sistema no tiene una intención, pero se puede observar que minimiza su energía y eso es algo bien definido. Al final, lo que busco es quizá una especie de coherencia colectiva más que de racionalidad. 
A partir del descubrimiento de la "racionalidad colectiva" algunos podrian concluir que es aceptable usar técnicas que sólo se ocupen de analizar el nivel agregado. Puesto que asociar el comportamiento de los individuos a macro patrones es tan dificil, dicho enfoque es analiticamente atractivo. ¿Qué diria de tal alternativa?

En realidad, esta alternativa está en el espíritu de la antigua macroeconomía, donde solíamos tener relaciones entre variables agregadas, y entonces se tienen cosas como los ciclos económicos de Goodwin (Goodwin 1951). No es ilegítimo pensar en términos de agregados. No necesariamente se tiene que estar interesado en explicar las relaciones agregadas en términos de individuos. Los banqueros centrales a menudo observan relaciones agregadas bastante simples sin preocuparse por qué fue lo que motivó a las personas; y ni siquiera intentan derivar las relaciones agregadas de modelos subyacentes. Para muchos propósitos (en particular para propósitos de política), centrarse en relaciones agregadas simples puede incluso ser mejor que preocuparse mucho por toda la mecánica de la economía. También se puede tener interés en la mecánica, pero para ciertos propósitos puede ser perfectamente legítimo querer relaciones entre variables agregadas. Para usar una metáfora familiar, no se tiene que entender la mecánica de un reloj para poder entender los movimientos regulares de sus manecillas.

Usted usa a menudo el término "emergencia" en sus trabajos. ¿̇ué quiere decir con eso? Entre las muchas interpretaciones de emergencia en filosofía (ver O'Connor y Wong 2009), al menos una, la interpretación de patrones irreducibles, parece implicar que no se podrian analizar algunas propiedades emergentes de nivel agregado en términos de las interacciones de unidades de un nivel inferior (p. ej., de los individuos).

Pero su estrategia parece ser exactamente esa. $\dot{\mathrm{C}}$ Por qué deberíamos esperar que el estudio de las interacciones a nivel individual sea una manera fructifera de analizar las propiedades de nivel agregado si estas últimas se consideran emergentes?

No soy filósofo, así que no sé mucho de estas cosas, pero si se mira hacia atrás, las personas que estaban en la interfaz -J. S. Mill, y quienes lo siguieron- estaban interesadas exactamente en esta distinción entre lo que ocurre en los diferentes niveles. Mi sensación primitiva, no filosófica, es que no se trata de una distinción entre observar a los individuos y luego observar las interacciones entre ellos. Lo que genera la diferencia en el nivel agregado es que los individuos interactúan 
entre sí. De modo que no puedo tomar ese individuo, examinarlo (la forma en que se comporta y sus decisiones) y concluir a partir de eso lo que hará la multitud. No puedo porque estoy eliminando la parte esencial que es la interacción. Diría que son las características individuales más su interacción las que generan la actividad aquí arriba, cuyas características son diferentes de las de los individuos específicos. Creo que lo que hace "irreducibles" los fenómenos agregados es el hecho de que no se puede derivar la propiedad agregada sumando el comportamiento de los individuos.

Un argumento importante -si no el argumento principal- de su libro reciente es que "la interacción directa entre agentes juega un papel crucial en la determinación de los resultados agregados" (Kirman 2011, 35). ¿Qué es tan "crucial" a ese respecto? ¿Es que la interacción directa es más "crucial" que otros elementos, p. ej., que las reglas de comportamientos de los propios agentes?

Bueno, cuando uno llega a la economía, al principio le dicen que se trata de la distribución de recursos escasos entre necesidades alternativas, o lo que sea; le dan una definición. Y uno dice, bueno, ¿cómo se logra esto? Bien, nos dicen, esto se logra porque las personas comercian entre sí y colectivamente eso lleva a resultados que tienen ciertas propiedades. Pero entonces uno dice: "comerciando entre sí, ¿cómo se organiza?” Y dicen: "Bueno, a través de un mercado. Allí hay algunos precios que están dados y luego todo el mundo usa esos precios”. Sí, pero ¿quién comercia con quién?

En el modelo estándar, la parte donde las personas se conocen, comercian, etc. - en la que suceden las cosas- simplemente falta. Tan pronto como se empieza a pensar en ello, uno se da cuenta de que, si las personas interactúan entre sí en los mercados, lo que hace una persona influirá en las demás. Por ejemplo, cuando conozco a alguien y me dice que está comprando un activo, eso probablemente influirá en lo que pienso de sus perspectivas. Toda esta interacción me parece importante, y sin embargo eso es algo que metemos bajo la alfombra en el esquema estándar. Hay muy pocos mercados donde el mecanismo de negociación real -la influencia real de una persona sobre otra- no es importante. Si se desea entender la actividad económica no se puede dejar de lado el hecho de que debe ocurrir entre socios comerciales.

Parece estar diciendo que es crucial que observemos las interacciones porque usualmente no aparecen en nuestra historia habitual de cómo funcionan las economias. Habría otra interpretación de "crucial", que sería algo como 
lo siguiente. Uno empieza con la proposición de los resultados agregados son determinados por las propiedades de los individuos -es decir, por las reglas que siguen-y por la estructura de sus interacciones. La estructura de interacciones sería "crucial" en el sentido de que el resultado agregado será más sensible a los cambios en la estructura de las interacciones que a los cambios en las reglas de comportamiento; cambiar las interacciones un poco modifica el resultado agregado, mientras que este resultado permanece igual para un amplio espectro de reglas de comportamiento. ¿También se suscribiría a esta interpretación de "crucial"?

Pienso que crucial tiene ambos significados. Lo que sabemos es que, si se cambian las reglas del mercado un poco, en realidad se puede hacer un gran cambio en el resultado. Déjeme darle un ejemplo muy simple. Tome un mercado de un activo financiero que está organizado como un libro de órdenes. Usted coloca sus órdenes y estas se borran cuando encuentran su contraparte, es decir, cuando el precio solicitado es menor que alguna oferta.

Una regla rige cuánto tiempo permanecen esas órdenes en el libro. ¿Permanecen simplemente un día y luego se eliminan? ¿Se dejan tres semanas? ¿Se dejan hasta que las personas que colocaron las órdenes las quitan? Aquí hay una regla organizativa simple que nada tiene que ver con las intenciones de la gente que coloca allí estas órdenes, pero que cambia mucho los precios reales. Cambiar en forma aparentemente menor las reglas de la organización puede cambiar realmente lo que sucede de manera considerable. De modo que lo que se permite que las personas hagan entre ellas, en vez de las reglas de comportamiento particulares que están siguiendo, puede llegar a ser muy importante.

En economía, la manera tipica de modelar las interacciones entre agentes sería usar teoría de juegos. Pero usted expresa cierta insatisfacción con la teoría de juegos. La teoría de juegos no es más flexible de como usted la describe? Sus criticas se aplican también a la teoría de juegos evolutivos, por ejemplo?

Si lee Ensayos sobre los fundamentos de teoría de juegos, de Binmore (1990), encontrará una sección donde dice que, infortunadamente, llegamos a una especie de callejón sin salida. Obtenemos esta regresión infinita ligada al problema del conocimiento común. Por ejemplo, conduzco con frecuencia de Aix a Marsella. Se tiene la autorruta y la vía paralela, la ruta nacional. Digamos que un día hay congestión en la autorruta y nadie en la nacional. Pienso: "Mañana tomaré la na- 
cional. Pero, espere un minuto, estos otros conductores también son inteligentes, así que mañana tomarán la nacional. Haría mejor ir por aquí. Pero, espere un minuto, estos conductores son muy inteligentes y pueden hacer lo mismo..." En realidad, no es lógicamente posible razonar sobre la solución de este tipo de problemas que en teoría de juegos se supone que las personas deben resolver.

Seguramente se puede definir un equilibrio, y decir que si estuviéramos allí nadie querría moverse. Pero entonces se llega al problema de cómo llegamos a este equilibrio; exactamente el mismo problema que tenemos con el equilibrio general.

Una salida es la teoría de juegos evolutivos, que no tiene personas razonando. Simplemente identifica a los individuos con estrategias, y las estrategias que lo hacen mejor se reproducen más, mientras que las estrategias que lo hacen peor desaparecen. Eso es sumamente mecánico; descarta todo razonamiento de parte de los individuos y, como dije antes se basa en una interpretación muy simplista de evolución.

Para ciertos problemas locales específicos, la teoría de juegos es una manera muy agradable de pensar cómo las personas podrían intentar resolverlos, pero tan pronto se enfrenta un problema general, como una economía o un mercado, pienso que es difícil creer que ocurre una interacción estratégica completa. Es pedirle demasiado a las personas. La teoría de juegos impone una enorme cantidad de razonamiento abstracto a las personas; mucho mayor que en la economía estándar, donde sólo se necesita conocer los precios y las propias preferencias.

Por eso pienso que la teoría de juegos, como enfoque de interacción a gran escala, probablemente no es el camino correcto. Pero todavía pienso que de la teoría de juegos proviene una idea realmente importante: tan pronto como las personas empiezan a preocuparse por el hecho de que lo que hacen tiene impacto en lo que hacen otras personas (y empiezan a pensar en ello), eso hace la vida muy diferente.

Está a favor de la modelación basada en agentes como método alternativo para estudiar las interacciones de los agentes. ¿ेPuede esbozar las características de este método?

Hay dos enfoques posibles para la modelación basada en agentes. $\mathrm{El}$ primero es comenzar con un modelo muy simple y rudimentario que se puede resolver analíticamente. Luego se generaliza y se simula este modelo más general. Conocemos los resultados analíticos del modelo simple y la pregunta es si estos resultados siguen vigentes en el modelo menos restrictivo. Ese enfoque se encuentra en el capítulo sobre mercados de pescado de mi nuevo libro (Kirman 2011, cap. 3). 
En el modelo simple con dos vendedores y muchos supuestos más restrictivos, examinamos analíticamente si las personas aumentan la probabilidad de acudir al vendedor del que obtuvieron los mayores beneficios en el pasado. Luego preguntamos qué pasaría si intentásemos generalizar el modelo. Como los resultados ya no se pueden derivar analíticamente, simulamos lo que sucede.

El enfoque alternativo - el enfoque de la vida artificial- da a las personas reglas muy arbitrarias para empezar, y les deja elegir reglas diferentes a medida que avanzan, y luego se ve si surge algo de eso. Ese fue el enfoque del mercado de valores de Santa Fe (p. ej., Arthur et al. 1997): arrojar a estos individuos en la olla y luego mirar la sopa y ver si algo ocurrió.

Una objeción a este segundo enfoque es que se tiene mucha libertad. Se pueden elegir de manera totalmente arbitraria las reglas básicas que se dan a las personas para empezar. David Colander en algún momento planteó esta objeción. Dijo que si se es razonablemente inteligente, se pueden dar las reglas correctas para obtener lo que se quiera.

Así, una objeción legítima a muchos modelos basados en agentes de hoy en día es que la especificación a menudo no es justificada; simplemente se ponen reglas que parecen intuitivas.

¿Para usted es entonces esencial tener una especie de justificación empirica?

No pido justificaciones sumamente estrictas - bien sean empíricas o teóricas- pero se necesitan algunas. Parece razonable suponer que las personas se apegan a reglas que parecen haber funcionado bien en el pasado. Si se comienza a poner reglas más específicas en el modelo, se querría alguna justificación empírica, idealmente. Y de nuevo, volvería a la misma idea: observar a los agentes económicos.

Por ejemplo, cuando la Unión Soviética colapsó, en Moscú surgieron todos esos quioscos alrededor de las estaciones del metro. Se tenía a todas esas personas que empezaban a comerciar por primera vez. Fuimos a este nuevo mercado y durante tres meses recogimos datos de los precios que cobraban y les preguntamos: “¿Cómo eligió la regla que usa? ¿Cómo decidió cambiar el precio?” Las respuestas fueron muy interesantes. Algunos vendedores dirían: "Bueno, miro el precio actual en Moscú". Otros decían: "Reviso el precio que pagué por los artículos cuando los obtuve, y luego añado un margen", o "trato de ajustarme al promedio de mi alrededor", o "intento abatir el precio más bajo de mi alrededor". Se tenían entonces todas estas reglas diferentes, luego hicimos pequeñas simulaciones y encontramos que se 
podían tener precios más altos en una estación del metro que en otra, no porque el ingreso de esta zona fuera mayor o menor, sino debido a la manera en que interactúan estas reglas. Nunca lo publicamos, pero era realmente interesante.

Según usted, ¿̇cuáles son las ideas más importantes que hasta ahora ha proporcionado en economía la modelación basada en agentes? ¿El trabajo de quién admira en particular?

Bueno, en primer lugar no me considero un modelador basado en agentes. Me parece interesante y pienso que es algo bueno que hacer, pero no me definiría como alguien dedicado a ese arte. Pero comencemos con personas como Bob Axtell y Josh Epstein. En su libro Crecimiento de sociedades artificiales (Epstein y Axtell 1996) se encuentra una hermosa ilustración de cómo se puedes usar estos métodos para explicar cosas que nos sería muy difícil explicar con modelos estándar. Desarrollaron lo que llamaron Sugarscape: un modelo computacional habitado por personitas que deambulan comiendo azúcar. Están programadas para que busquen donde hay más azúcar, pero debido por supuesto a que todas terminan yendo y consumiendo en los mismos lugares, allí hay menos azúcar, y siguen adelante. La sociedad se organiza a sí misma y se puede ver que ocurren ciclos: aquí crece más azúcar, la comen y luego todas continúan, y después regresan cuando el azúcar vuelve a crecer. Se obtienen estos ciclos y patrones emergentes que no se podrían haber generado con un modelo de tipo ordinario.

Tenemos entonces algunos buenos modelos que desarrollan este tipo de ideas para tratar la emergencia de autoorganización colectiva. Pero no creo que hayamos visto muchos modelos de mercado, muchos modelos económicos, que tengan un resultado tan impactante. Por el momento, en cierto modo no hemos dado el salto. Sin embargo, tenemos algunos buenos ejemplos, como los modelos de Schelling (Schelling 1978). ¿Cuál fue la motivación de Schelling? Mostrar que lo que sucede a nivel macro es muy diferente de lo que sucede a nivel individual. Para mí, ese fue entonces un temprano ejemplo de modelación basada en agentes, y un hermoso ejemplo. Como se puede ver desde el comienzo de mi libro, soy admirador de Schelling.

Leigh Tesfatsion es una persona que ha hecho un gran esfuerzo para reunir gente en torno de la modelación basada en agentes (p. ej., Tesfatsion y Judd 2006). Por tanto, ella debería obtener mucho crédito por impulsar este campo. Blake LeBaron es otra; un economista de Brandeis que trabaja sobre mercados financieros. Ha hecho algunos modelos muy buenos basados en agentes en los que logra reproducir 
fenómenos realmente difíciles de producir con modelos estándar para series de precios de acciones (p. ej., LeBaron et al. 1999). También se puede considerar basado en agentes parte del trabajo que hicieron Buzz Brock y Cars Hommes. De nuevo, esas son personas que realmente desarrollan modelos interesantes; tienen fundamentos teóricos pero también usan simulaciones.

No soy un gran admirador de las personas que arman un modelo y luego simplemente giran la palanca. Los que sólo dicen: "Ahora inspeccionaré todo el espacio de parámetros para ver qué puede suceder y qué no sucede". No creo que esto sea perspicaz. Pero hay personas que realmente tienen una visión. Para volver a la emergencia, descubren que han generados interesantes fenómenos emergentes a partir de modelos simples basado en agentes.

Algunos académicos parecen creer que la modelación basada en agentes ofrece una herramienta poderosa para el análisis de politica. Por ejemplo, Robert Axtell dice: "Con esa máquina llena de agentes se puede averiguar qué procesos dan los datos empiricos sobre la distribución del ingreso real y la riqueza. Luego se pueden mover tres años y ver qué ocurre. Pienso que es un nueva manera de hacer macroeconomia; es una nueva manera de hacer politica" (entrevistado en Colander 2004, 285). ¿Qué tipo de preguntas de politica cree que puede abordar la modelación basada en agentes?

Cuando di una charla en el Banco de Inglaterra, uno de los directores, Spencer Dale, dijo: "Me gusta lo que haces, pienso que es interesante, pero ya sabes, tengo que hacer política monetaria. ¿Qué voy a decir cuando estemos sentados tratando asuntos de política cotidiana? Es mucho más fácil con los modelos regulares porque sabemos lo que se supone que debemos decir y sabemos los que las personas esperan que digamos y podemos hablar de eso. Pero si quieres que use lo que dices, debes explicarme cómo hago el tránsito".

Creo que aún no estamos listos para eso. Por supuesto, mi opinión es que la macroeconomía ortodoxa tampoco está lista para eso, es solo que estamos acostumbrados a hacerlo así.

Aunque hay algunos casos donde se puede empezar a ver cómo se pueden usar estos modelos para guiar la política. Bridget Rosewell, qué ahora es la Asesora Económica Jefe del London Council en Reino Unido, me pidió que le ayudara a ver qué pasaría si hubiese un mercado de permisos de contaminación. Una manera de hacerlo era intentar establecer un modelo teórico simple. Pero la alternativa era averiguar cómo se comportarían las personas en ese mercado; considerar qué estrategias se podían usar, y luego simular eso y ver 
que salía de eso. Con Nick Vriend, encontramos que cambiando las reglas que usan los agentes podíamos cambiar mucho la volatilidad de los precios resultantes. Eso era lo que preocupaba al gobierno en ese momento, es decir, que estos precios iban a ser demasiado volátiles. Observando el modelo basado en agentes, encontramos que podríamos reducir sustancialmente la volatilidad al no permitir que las personas hicieran ciertas cosas.

Hay entonces algunas áreas en las que se usan estos modelos, pero decir que ahora debemos pasar a hacer política macroeconómica con base en la modelación basada en agentes es demasiado. Hay algunos optimistas como Joe Stiglitz, Mauro Gallegati y otros que están empezando a hacer recomendaciones macroeconómicas sobre esta base. Pero otras personas no están tan convencidas.

${ }_{\dot{\zeta}}$ el trabajo de Andy Haldane del Banco de Inglaterra? Parece que él intenta usar este tipos de técnicas para entender mejor qué significa "demasiado grande para quebrar", lo que por el momento parece un eslogan vacio?

Ese es exactamente el tipo de cosas que tiene en mente. Y usted tiene rezón en que en este momento "demasiado grande para quebrar" es solo un eslogan. Escuchamos que si este banco se hundía, los demás también se hundirían, por lo que no deberíamos dejar que el primero se hundiera. Pero eso no está articulado de la manera rigurosa que se requeriría de alguien que hace economía matemática. La pregunta es, ¿en qué punto esa interdependencia se vuelve tan importante en la red que no se puede dejar que uno de nuestros nodos se caiga? Podemos observar una red bancaria y considerar si dejar que este nodo decaiga derribará a todo el sistema con él. El paso que debemos dar ahora no es sólo mirar el tamaño de estos nodos sino preguntar ¿cuáles son los enlaces? ¿Cuáles son los arreglos que existen entre estos bancos? ¿Qué pasa si un banco de veras se mete en problemas? ¿Los arreglos contractuales son tales que la autoridad supervisora vería que todo el sistema se meterá automáticamente en problemas o son tales que la autoridad podría quedarse a un lado? Si no tenemos la respuesta a ese tipo de pregunta, entonces no sabemos si el problema se propagará. Pienso entonces que el siguiente paso es estudiar de cerca los mercados, y eso es lo que pueden hacer los bancos centrales porque tienen los datos: saben hasta qué punto Barclays está vinculado con HSBC, y así sucesivamente. Una vez sepamos más sobre lo que son estos enlaces, podemos conectarlos como nodos y enlaces. Debemos estudiar estos enlaces y nodos antes de que podamos decir si es probable que esta red colapse. 
En resumen, algo semejante a la resiliencia sería una dimensión politica a la que pueden contribuir la teoría de redes y la modelación basada en agentes.

Sí, y la resiliencia de la red tiene más importancia que la resiliencia de los bancos individuales. Solíamos decir, "pongamos estas restricciones a los bancos individuales", pero si sólo nos preocupan los bancos individuales no sabemos qué significa "demasiado grande para quebrar", porque "demasiado grande para quebrar" se refiere realmente al impacto sobre otros. No significa lo qué le ocurre a uno significa lo que lo que le ocurre a los otros. Poner restricciones a los individuos solo significa que no queremos que nadie quiebre.

Continuando con asuntos de politica, volvamos al problema planteado por David Colander que usted mencionó antes. Él expresa cierto escepticismo sobre la capacidad de la modelación basada en agentes para ofrecer consejos claros de politica: "Cuando se tienen modelos con equilibrios múltiples, dependencia de la trayectoria, dinámica no lineal, gustos endógenos, restricciones institucionales e histéresis, hay tantos grados de libertad que la teoría ofrece poco en materia de restricciones a la politica. Con tantos grados de libertad, un modelador suficientemente capaz puede idear un modelo teórico para apoyar cualquier politica" (Colander 2003, 20-21). En su opinión, ¿̇cuáles son las perspectivas del análisis de politica basado en agentes? ¿Cree que podemos reunir suficiente evidencia para restringir las especificaciones de nuestro modelo?

Al final, las personas que construyen estos modelos deberían poder ver cuáles de los supuestos impulsan los resultados. En general, no es cierto que todos los supuestos tengan igual peso. Algunos pueden cambiar sin mucho impacto. De modo que al final deberíamos empezar a ver que supuestos están impulsando un resultado particular.

Déjeme darle un ejemplo muy simple. Muchas personas intentan reproducir las distribuciones exponenciales o las distribuciones tipo Pareto de toda gama de fenómenos, bien sean los precios bursátiles, el tamaño de las firmas, el tamaño de las ciudades o lo que sea. Esta distribución aparece en todas partes; es asombroso. Ahora bien, si se observa cuidadosamente lo que sucede aquí, resulta que siempre se basa en la siguiente observación: si se toma el caso de las ciudades, lo que se hace es decir que cuando alguien quiere mudarse a una ciudad, la probabilidad de hacerlo es proporcional al tamaño de la ciudad existente. Con esa regla, automáticamente se produce una distribución de Pareto de tamaños de ciudad. Esa misma reflexión está detrás de los 
modelos macro de distribución del capital. Lo que sucede es que las firmas que tienen más capital son aquellas que obtendrán más capital.

Vemos estas maravillosas distribuciones de Pareto en todas partes, pero de hecho se deben a un mecanismo subyacente muy simple. Creo que lo mismo ocurrirá en otro lado. No es verdad que solo porque hay tantas de estas cosas nunca veremos la luz; empezaremos a ver qué supuestos estén impulsando los resultados. Y una vez lo hagamos eso hará mucho más fácil nuestra tarea.

En su libro reciente se encuentran muchos términos como "entender" $y$ "explicar". En cambio, rara vez usa términos como "predecir", "pronosticar", "controlar", "intervenir" y "diseñar politicas". ¿Qué le responderia a alguien que cree que la economía es principalmente el negocio de hacer predicciones y recomendaciones de política y que, si bien su empeño es quizá valioso para propósitos explicativos, es de poca utilidad en la buisqueda de esos objetivos?

Mi esposa me dice lo mismo. Me dice: "Cada vez que hablas, siempre tengo la sensación de alguien que observa un nido de hormigas o una colmena y está muy interesado en lo que está ocurriendo allí y siente mucha curiosidad, pero no está preocupado particularmente por hacer que funcione mejor. En cierto sentido, eres un observador curioso y no alguien que se dedique realmente a hacer algo". Creo que esa es una crítica legítima. No sé si esa es mi naturaleza o qué. Sólo encuentro estas cosas muy interesantes. Razono un poco como un entomólogo.

Sobre el pronóstico; si se cree en este tipo de enfoque de sistemas, pronosticar es un ejercicio muy difícil. Observe a las personas que hacen pronósticos: tienen estos modelos grandes y muy sofisticados. Pero, si se fija en las discusiones sobre cuál será la tasa de crecimiento en la Unión Europea o la tasa de crecimiento en Estados Unidos, por ejemplo, verá cuán rápidamente se revisan estas cosas. De un mes al siguiente, el gobierno francés dice: "Bueno, bajamos del 2,5 al 1,7". ¿No es ese un gran cambio? Es, de hecho, un cambio muy grande; hace una gran diferencia en términos de lo que deberíamos hacer con el déficit, y así sucesivamente.

Creo que lo haremos mucho mejor observando la naturaleza de la evolución en vez de intentar predecir "esto va a suceder"; tratando de decir, ¿este tipo de cambio que está sucediendo llevará a una evolución más positiva o a una evolución más negativa? Eso es algo sobre lo que probablemente podamos decir algo, pero decir que el crecimiento del PIB será de 1,2, de 2,3 o lo que sea, creo que no estamos en ese juego.

Creo que al usar estas cosas para hacer recomendaciones de política uno debe ser bastante modesto. De hecho, no estoy seguro de cuánto 
tiene que ver realmente con el uso de la economía y cuánto con tener una visión del mundo. Cuando Hugo Sonnenschein me dijo que la macroeconomía tenía que ver con la sabiduría, bueno, he llegado a pensar cada vez más que él quizá tenía razón. En cierto sentido, la macroeconomía es mucho más sobre la experiencia y bastante menos sobre el análisis formal. Pero eso es solo una opinión personal, y una especie de excusa: una manera de decir: “iLo siento, queridos muchachos, No puedo hacer esto!"

También queremos preguntarle sobre sus sentimientos hacia el uso de las matemáticas en economia. Muchos economistas heterodoxos argumentan que se esperan demasiadas matemáticas de los economistas, y que la profesión se ha obsesionado por ser excesivamente formal. ¿Cómo cree que los economistas deberían usar las matemáticas?

Me parece extraño que debamos preocuparnos por una herramienta, que esta herramienta de alguna forma deba ser un criterio para juzgar el trabajo o ser objeto de crítica. Las matemáticas son simplemente una forma de simplificar un problema, quizá erróneamente a veces. John Chipman hizo una revisión de la teoría del comercio internacional hasta cierto momento, que se publicó en Econometrica (Chipman 1965), y allí dice que a veces las matemáticas resultan útiles porque permiten enmarcar las cosas de manera más clara. Para él, resolver ciertos problemas de economía sin matemáticas es como cruzar el Canal a nado. Es una hazaña admirable y todos la aplauden, pero quizá no sea la manera más fácil de cruzar el Canal. Entonces, en cierto sentido, no me parece que tenga sentido evitar las matemáticas por principio. Pero obsesionarse con las matemáticas tampoco parece tener sentido.

De hecho, no creo que el problema fundamental tenga que ver con matemáticas y no matemáticas. Las matemáticas son solo una herramienta, y en realidad cualquier herramienta que se encuentre alrededor, bueno, está bien. Pero en cierto modo ahora hay una jerarquía, y se cree que hacer matemáticas es una cosa superior. Hace poco, en nuestro grupo, rechazaron a una estudiante de doctorado que yo quería que aceptaran porque no tenía sólida formación en matemáticas. Y ella había hecho cursos de negocios, etc., y quería trabajar en finanzas del comportamiento. Pero dijeron: "No es una persona seria”. En mi opinión, este es un mal criterio, porque para que haga algún progreso en este problema particular no requiere necesariamente que sea matemática. Hay mucha gente, como Akerlof 
y Bob Shiller, que no usa matemáticas de alta potencia, pero tiene buenas ideas.

Muchos de mis colegas piensan que Schelling no debería haber ganado el premio Nobel. Cuando les pregunto por qué, dicen: “¡casi no hay matemáticas en lo que hace!"Y es cierto que no usa matemáticas difíciles. Pero tiene ideas difíciles y realmente interesantes. ¿Por qué juzgarlo por las herramientas matemáticas que usa?

Hace años participé en la organización de conferencias con Cristopher Zeeman, uno de los fundadores de la teoría de catástrofes y jefe del departamento de matemáticas de la Universidad de Warwick. Él solía organizar rencontres entre matemáticos y personas de otras disciplinas, y organizamos uno entre economistas y matemáticos. Teníamos varios grandes matemáticos - John Milnor, Steve Smale, Rene Thom- y otros matemáticos maravillosos. Y en el otro lado, a Gérard Debreu, Hugo Sonnenschein, Werner Hildenbrand y un grupo de economistas matemáticos muy distinguidos. Luego de las dos o tres horas primeras, creo que fue Milnor quien dijo: “Todos sabemos que pueden hacer matemáticas, no tienen que demostrarlo. Cada quien hace lo suyo. Quieren mostrarnos que son buenos para hacer cierto tipo de matemáticas; eso está bien. Pero estamos interesados en los problemas económicos. Pensábamos que iban a hablarnos de problemas económicos y que nosotros íbamos a usar nuestras herramientas matemáticas para ayudarles. Pero todo lo que nos dicen es sobre las herramientas matemáticas que usan y cómo les va de bien con ellas. Pero eso no lleva a crear mucho". Creo que tenía toda la razón. Después de eso, los economistas quedaron en silencio y empezaron a moverse incómodos en sus asientos. De todos modos, Debreu nunca decía mucho, pero era claro que se sentía insultado, porque le gustaba considerarse a sí mismo como un matemático.

\section{¿Puede resumirnos su diagnóstico del estado de la macroeconomía?}

Diría que la teoría macroeconómica ha llegado a un callejón sin salida en el sentido de que nos aferramos a un modelo particular: el equilibrio general. Pero no es en realidad equilibrio general, quiero decir: $j$ es el modelo de un solo individuo! En particular, se ha vuelto matemáticamente sofisticado sin representar las características fundamentales de la macroeconomía. Así, diría que personas como Kydland y Prescott, y así sucesivamente, personas así, merecen sus premios Nobel porque cambiaron la forma de hacer macroeconomía. Pero en mi opinión no fue un cambio positivo. Pienso que dejamos de preocuparnos por la macroeconomía como un sistema con interdependencia, etc., y nos 
obsesionamos con esta visión particular de cómo funciona. Una idea predominante es la de choques externos, y en particular la idea de que los choques que le ocurren a la economía deberían ser esencialmente choques tecnológicos. Como dijo Joe Stiglitz, ¿qué entenderíamos por un choque tecnológico negativo? ¡Que la gente olvida lo que podían hacer antes?

Así que tenemos la idea de que hay un sistema que está en equilibrio y que de vez en cuando se sale del equilibrio por "un choque". ¡Pero los choques son parte del sistema! Hemos seguido un camino que realmente no nos permite decir mucho sobre los movimientos reales e importantes de la macroeconomía. Al final, deberíamos estar más interesados no en los periodos en los que la economía avanza con relativa suavidad sino en los periodos en los que cambia. La gente suele decir: "Bueno, este no es un periodo normal, y analizamos lo que sucede en periodos normales, y todo esto son desviaciones de eso". Pero deberíamos estudiar los periodos no normales, en vez de los normales, porque eso es lo que causa problemas reales. Y no hacemos eso.

De modo que mi visión del estado de la macroeconomía es que en cierto modo tiene una visión errónea: una visión de equilibrio y una visión de estado estacionario. Pero lo que es importante e interesante e macroeconomía es justamente cuando esas dos cosas no se mantienen. ¿Cómo se puede hablar de equilibrio cuando pasamos de un desempleo del 5\% a un desempleo del 10\%? Si se está en Chicago, se dice: "Bueno, ese $5 \%$ adicional calculó que le era mejor estar sin trabajo". Pero veamos la realidad; eso no es lo que ocurre. Las personas no quieren estar sin trabajo. Para ellas es una tragedia; afecta su identidad. Me molesta mucho pensar que la gente sólo diga: "Ah, sólo otro 5\%; manejamos esto muy bien”. Millones de personas están sin trabajo, ¿y eso no nos preocupa?

Esa es la principal falla de la macroeconomía. No aborda los graves problemas que enfrentamos cuando salimos del equilibrio. Y está fuera del equilibrio la mayoría del tiempo.

Ese fue el diagnóstico, ¿̇qué tratamiento propondría usted? ¿̇ cómo piensa que se debería reformar la profesión?

Ah, la profesión. Bueno, como dice Buzz Brock, deberíamos abrir nuestra mente, pero no tanto como para que se nos caiga el cerebro. Creo que deberíamos tener en cuenta todo tipo de enfoques diferentes de la macroeconomía y observar cómo funcionan los mercados. Deberíamos empezar a incorporar evidencia empírica, en vez de 
obsesionarnos con modelos sumamente limitados. Deberíamos tratar de seguir pensando en todas estas cosas que impactan la economía y ver si podemos incorporar algunas de ellas, y quizá descartar algunas otras. No deberíamos centrarnos completamente en producir un modelo cerrado que se pueda resolver y luego decir que es una representación de lo que vemos allí afuera. Si no se mira allí afuera, este modelo siempre estará separado de la realidad.

Esa es una crítica de la profesión. Si se quiere tener éxito, se tiene que publicar en buenas revistas. Lo que publican las buenas revistas son básicamente avances de trabajos previos. Eso es absolutamente razonable, y es comprensible que la profesión tenga mucha inercia. Pero tampoco debería estar tan encerrada que cualquier cosa que sea más innovadora no pueda entrar en las principales revistas. Creo que si se revisa American Economic Review, no es tan mala, pues incorpora bastante economía del comportamiento, economía experimental, etc. Pero creo que en macroeconomía es sumamente conservadora. Si se produce un modelo que no concuerda con lo que se estaba haciendo antes, es mucho difícil publicarlo.

Un joven economista, creo que estaba en la UCLA, que me escribió cuando publiqué un artículo titulado ¿A quién o a qué representa el agente representativo? (Kirman 1992). Me dijo: "Estimado profesor, en realidad estoy de acuerdo con lo que dijo. Creo que es del todo correcto intelectualmente. Lamentablemente, soy un joven macroeconomista que es profesor asistente. Construyo modelos basados en un agente representativo. Sé cómo hacer eso, y sé cómo publicar eso. Y necesito la titularidad. Una vez tenga la titularidad, quizá pueda dar el giro y empezar a pensar en el tipo de modelos que no utilizan al agente representativo, pero infortunadamente, lo que creo que pasará es que para entonces ya habré adquirido el hábito de hacer eso. Publicaré mis artículos, obtendré buena reputación, conseguiré una promoción y tal vez nunca vuelva a pensar en esto. Pero de todos modos ¡muchas gracias por la idea!"

¡Eso es algo deprimente!

¡No! Pensé que era sumamente honesto. Así es como son las cosas. Es muy difícil. Es curioso que en lugares como el Journal of Political Economy se acepten modelos tipo Schelling, etc., de vez en cuando. Entonces no es cierto que la profesión sea un bloque sólido contra cualquier cosa innovadora. Pero hay una sospecha natural contra cosas que no se pueden reducir a un equilibrio estándar. 
Entonces, ¿qué reformas son necesarias? Bueno, la manera de reaccionar de la profesión es crear nuevas revistas, ¿cierto? Por ejemplo, desde el principio estuve involucrado en el Journal of Economic Behavior and Organization, una revista a la que se consideraba marginal y rara. Pero hoy es una revista muy aceptable; está bien pensada. Esa es la forma en que ocurrirán estos cambios. Me gustaría creer en una selección natural pura, pero pienso que hay mucha inercia en la profesión.

De vez en cuando me invitan a conferencias de neurobiología. Allí las personas están realmente interesadas en lo que están haciendo. Una vez empiezan a contar lo que están haciendo, no se las puede detener. Pero en la discusión en conferencias de economía, usualmente se trata de quién va a conseguir una promoción y dónde, de quién publicó en esta o aquella revista. Deberíamos cambiar eso.

Si se va a una conferencia de economía experimental, es diferente. Están más entusiasmados con lo que hacen. Pero si se va a una conferencia de macroeconomía, ¡todo lo que se quiere hacer es salirse! Es el precio se debe pagar. Hay que estar allí. Todos están allí, escuchándose muy seriamente entre sí.

\section{$\dot{¿}$ En últimas, es optimista o pesimista sobre el futuro de la profesión?}

Bueno, como podría haber dicho Keynes, creo que "en el largo plazo" las cosas mejorarán mucho. Creo que las personas se darán cuenta de que la economía es un tema maravilloso y emocionante y dejarán de tratarla como un ejercicio analítico independiente de la realidad. Muchas personas hacen trabajo empírico, por supuesto, pero no un trabajo empírico imaginativo y emocionante. Suele ser muy rutinario. Pero tengo más simpatía por la persona que empieza a estudiar y a analizar datos de un mercado particular -el mercado del trigo o algo así- y realmente ve cómo funciona, que la que siento por la persona que construye el modelo DSGE de enésima generación. Creo que la primera trata de entender lo que está ocurriendo. Las herramientas que usa quizá no sean súper emocionantes pero aumenta nuestra comprensión del mundo económico. De eso se trata. Eso es lo que pensé cuando llegué a la economía. Pensé: "esto se trata realmente de entender cómo suceden los fenómenos económicos. Qué tema tan maravilloso y emocionante".

\section{REFERENCIAS BIBLIOGRÁFICAS}

Arthur, W. Brian, John Holland, Blake LeBaron, Richard Palmer, and Paul Tayler. 1997. Asset pricing under endogenous expectations in an 
artificial stock market. In The economy as an evolving complex system II, eds. W. Brian Arthur, Steven N. Durlauf, and David A. Lane. Reading (MA): Addison-Wesley, 15-44.

Binmore, Kenneth G. 1990. Essays on the foundations of game theory. Cambridge (MA): Basil Blackwell.

Chipman, John S. 1965. A survey of the theory of international trade: part 1, the classical theory. Econometrica, 33(3): 477-519.

Colander, David. 2003. Post Walrasian macro policy and the economics of muddling through. International Journal of Political Economy, 33(2): 17-35.

Colander, David, Richard P. F. Holt, and J. Barkley Rosser, Jr. 2004. The changing face of economics: conversations with cutting edge economists. Ann Arbor: University of Michigan Press.

Churchland, Patricia S., and Terrence J. Sejnowski. 1992. The computational brain. Cambridge (MA): MIT Press.

Debreu, Gérard. 1974. Excess demand functions. Journal of Mathematical Economics, 1(1): 15-21.

Epstein, Joshua M., and Robert L. Axtell. 1996. Growing artificial societies: social science from the bottom up. Cambridge (MA): MIT press.

Föllmer, Hans. 1974. Random economies with many interacting agents. Journal of Mathematical Economics, 1(1): 51-62.

Goodwin, Richard M. 1951. The nonlinear accelerator and the persistence of business cycles. Econometrica, 19(1): 1-17.

Gordon, Deborah M. 2010. Ant encounters: interaction networks and colony behavior. Princeton: Princeton University Press.

Härdle, Wolfgang, and Alan P. Kirman. 1995. Nonclassical demand: a model-free examination of price-quantity relations in the Marseille fish market. Journal of Econometrics, 67(1): 227-257.

Hildenbrand, Werner. 1994. Market demand: theory and empirical evidence. Princeton: Princeton University Press.

Hildenbrand, Werner, and Alan P. Kirman. 1976. Introduction to equilibrium analysis: variations on themes by Edgeworth and Walras. Amsterdam: North Holland Publishing Co.

Hildenbrand, Werner, and Alan P. Kirman. 1988. Equilibrium analysis: variations on themes by Edgeworth and Walras. Amsterdam: NorthHolland Publishing Co.

Kirman, Alan P. 1992. Whom or what does the representative individual represent? The Journal of Economic Perspectives, 6(2): 117-136.

Kirman, Alan P. 2011. Complex economics: individual and collective rationality. Londres: Routledge.

Kirman, Alan P., and Miriam Tesch1. 2010. Selfish or selfless? The role of empathy in economics. Philosophical Transactions of the Royal Society B: Biological Sciences, 365(1538): 303 -317.

LeBaron, Blake, W. Brian Arthur y Richard Palmer. 1999. Time series properties of an artificial stock market. Journal of Economic Dynamics and Control, 23(9): 1487-1516.

Mantel, Rolf R. 1974. On the characterization of aggregate excess demand. Journal of Economic Theory, 7(3): 348-353. 
Morishima, Michio. 1984. The good and bad uses of mathematics. In Economics in disarray, eds. Peter John de la Fosse Wiles, and Guy Routh. Oxford: Basil Blackwell, 51-73.

O'Connor, Timothy, and Hong Yu Wong. 2009. Emergent properties. The Stanford Encyclopedia of Philosophy, ed. Edward N. Zalta. http:// plato.stanford.edu/archives/spr2009/entries/properties-emergent/

Oullier, Olivier, J. A. Scott Kelso, and Alan P. Kirman. 2008. Social neuroeconomics: A dynamical systems perspective. Revue d'économie politique, 118(1): 51-62.

Schelling, Thomas C. 1978. Micromotives and macrobehavior. Nueva York: W.W. Norton \& Company, Inc.

Sonnenschein, Hugo. 1972. Market excess demand functions. Econometrica, 40(3): 549-563.

Tesfatsion, Leigh, and Kenneth L. Judd. 2006. Handbook of computational economics: agent-based computational economics. Amsterdam: NorthHolland Publishing Co.

Vinković, Dejan, and Alan Kirman. 2006. A physical analogue of the Schelling model. Proceedings of the National Academy of Sciences, 103(51): 19261-19265. 